\title{
Maintaining the mucosal barrier in intestinal inflammation
}

The intestine is a complex environment at the interface between the host, pathogens and commensal microorganisms. Two new papers published in Cell now describe how microbial metabolites, IL-18 and the NLRP6 inflammasome have key roles in barrier function during homeostasis and intestinal inflammation.

An important component of intestinal homeostasis and inflammation is the integrity of the intestinal barrier, a physical barrier integrating the epithelial production of mucus and antimicrobials with effector functions of the mucosal immune system. Previous work had shown that IL-18 is a key mediator of intestinal homeostasis (especially in the host-microbiota interface), but also had proinflammatory actions. As such, the new research sought to determine the role of IL-18 in both steady-state conditions and during inflammatory disease.

In the first study, Levy et al. explored the relationship between the host-microbiota interface and NLRP6 inflammasome signalling. Using a series of knockout and/or germ-free mice, the researchers demonstrated that the gut microbiota is critically important for and modulates NLRP6 inflammasome activation and downstream secretion of IL-18 and antimicrobial peptides (AMPs). Conversely, the inflammasome-AMP axis influences the composition and stability of the normal gut microbiota; inflammasome-deficient mice have dysbiosis and an altered gut microbiota compared with wild-type mice.

Using a combined metagenomics and metabolomics approach, the investigators found that specific microbiota metabolites modulate NLRP6 inflammasome signalling and antimicrobial pathway activation (IL-18 and AMP secretion) - taurine was identified as an activator of the mucosal inflammasome, whereas spermine and histamine were found to be inflammasome inhibitors.

Crucially, administration of taurine, spermine or histamine to the mice (via their drinking water) led to compositional changes in the gut microbiota and profound effects on host physiology and disease susceptibility in an inflammasomedependent manner. Compared with controls, taurine-treated mice had improved weight loss, reduced colitis severity, enhanced survival and improved mucosal barrier integrity after induction of colitis via dextran sodium sulfate (DSS) administration. By contrast, colitis was exacerbated in mice treated with histamine.

In the second study, Nowarksi et al. focused on the role of IL-18 in the maintenance of the intestinal barrier during homeostasis and intestinal inflammation. Conditional knockout mice lacking either IL-18 or the IL-18 receptor were generated to try and delineate the role of IL- 18 .

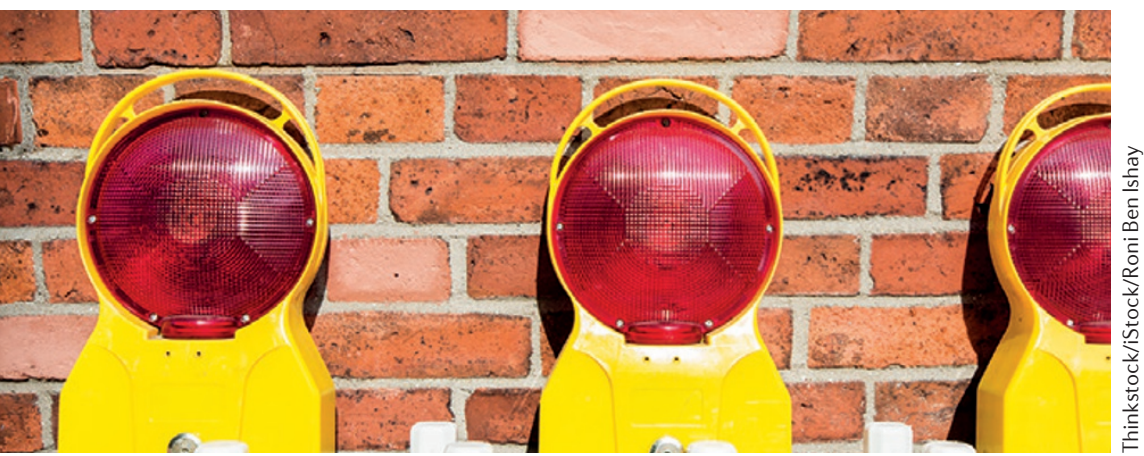

The researchers found that epithelial IL-18-IL-18 receptor signalling promoted DSS-induced colitis in mice. Deletion of $I l 18$ or its receptor, $I l 18 r 1$, specifically in intestinal epithelial cells protected mice from DSS-induced colitis and mucosal damage. By contrast, deletion of Il18bp (a negative regulator of IL-18) resulted in hyperactive IL-18 signalling and led to severe colitis (extensive bleeding and intestinal tissue damage) upon administration of DSS, which was associated with loss of mucus-producing goblet cells.

Further experiments in mice showed that an increased level of IL-18 was a critical factor in disrupting barrier function. Hyperactive IL-18 signalling seemed to prevent the maturation of goblet cells prior to the development of colitis and onset of DSS-induced symptoms. Moreover, daily injections of IL-18 in wild-type mice increased disease severity in DSS-induced colitis. On a transcriptional level, expression of goblet cell differentiation or maturation factors (Gfi1, Spdef and Kif4) were inhibited by increased IL-18 (in Il18bp-knockout mice or after IL-18 injection in wild-type mice).

Findings from these two new papers highlight the complexity of inflammatory disorders such as IBD, which exists at the junction between genetics, immune response, gut microbiota and environment. Both sets of researchers hope to unravel further mechanistic insights as a follow-up to these studies. They are hopeful that their findings have pinpointed future targets or treatment approaches for intestinal inflammation either by targeting key cytokines directly (IL-18) or by manipulating microbial metabolites as a 'postbiotic' approach.

Katrina Ray

ORIGINAL ARTICLES Levy, M. Microbiotamodulated metabolites shape the intestinal microenvironment by regulating NLRP3 inflammasome signalling. Cell 163, 1428-1443 (2015) | Nowarski, R. Epithelial IL-18 equilibrium controls barrier function in colitis. Cell 163. 1444-1456 (2015)

FURTHER READING de Souza, H. S. P. \& Fiocchi, C. Immunopathogenesis of IBD: current state of the art. Nat. Rev. Gastroenterol. Hepatol. http://dx.doi.org/10.1038/nrgastro.2015.186 